Document downloaded from:

http://hdl.handle.net/10251/61072

This paper must be cited as:

Carrascosa Casamayor, C.; Klugl, F.; Ricci, A.; Boissier, O. (2015). From Physical to Virtual: Widening the Perspective on Multi-Agent Environments. En Agent Environments for MultiAgent Systems IV. 4th International Workshop, E4MAS 2014 - 10 Years Later, Paris, France, May 6, 2014. 133-146. doi:10.1007/978-3-319-23850-0_9.

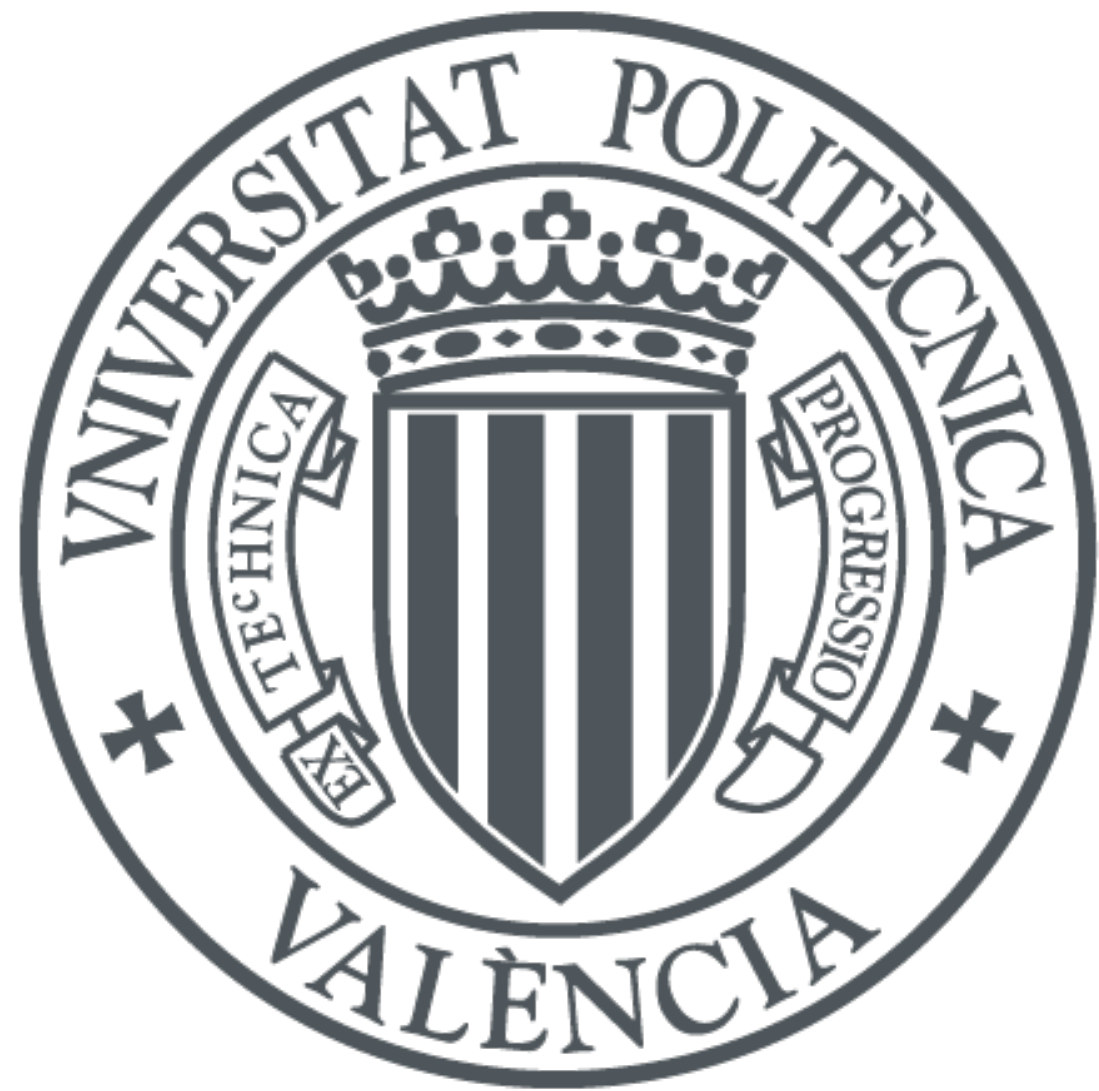

The final publication is available at

http://link.springer.com/chapter/10.1007\%2F978-3-319-23850-0_9

Copyright

Additional Information

The final publication is available at Springer via http://dx.doi.org/10.1007/978-3-319-238500_9 


\title{
From Physical to Virtual: Widening the Perspective on Multi-Agent Environments
}

\author{
C. Carrascosa ${ }^{1}$, F. Klügl ${ }^{2}$, A. Ricci ${ }^{3}$ and O. Boissier ${ }^{4}$ \\ 1 Universitat Politècnica de València, \\ DSIC, Camino de Vera sn 46022 Valencia, Spain \\ carrasco@dsic.upv.es \\ 2 School of Natural Science and Technology \\ Örebro University \\ 70182 Örebro (Sweden) \\ franziska.klugl@oru.se \\ 3 DISI, Alma Mater Studiorum - Università di Bologna \\ Via Sacchi 3, Cesena, Italy \\ a.ricci@unibo.it \\ 4 FAYOL - ENS Mines \& Laboratoire Hubert Curien CNRS:UMR 5516, \\ Saint-Etienne, France \\ boissier@emse.fr
}

\begin{abstract}
Since more than a decade, the environment is seen as a key element when analyzing, developing or deploying Multi-Agent Systems (MAS) applications. Especially, for the development of multi-agent platforms it has become a key concept, similarly to many application in the area of location-based, distributed systems. An emerging, prominent application area for MAS is related to Virtual Environments. The underlying technology has evolved in a way, that these applications have grown out of science fiction novels till research papers and even real applications. Even more, current technologies enable MAS to be key components of such virtual environments.

In this paper, we widen the concept of the environment of a MAS to encompass new and mixed physical, virtual, simulated, etc. forms of environments. We analyze currently most interesting application domains based on three dimensions: the way different "realities" are mixed via the environment, the underlying natures of agents, the possible forms and sophistication of interactions. In addition to this characterization, we discuss how this widened concept of possible environments influences the support it can give for developing applications in the respective domains.
\end{abstract}

\section{Introduction}

The last decade has seen the increase of the different kinds of environment that Multiagent Systems (MAS) have been applied to/in, due to the increase in numbers and complexity of the application domains as well as their diversification. One category of these environments with a huge development is related to Virtual Environments (VE), proposing more and more sophisticated and credible 
simulations of some "reality" for one or more user to be immersed into. Agent technologies have been also proposed for supporting interaction for humans in such environments [13]. VEs are not restricted to traditional virtual reality application domains such as art, entertainment or education, but there is a variety of other sophisticated opportunities for mixing physical, virtual, simulated, etc. With the tremendous development of related technologies, intelligent agents may interact with other agents or with humans in environments in a way far beyond the MAS-environments discussed in the E4MAS community before.

In fact, it is easy to see that current developments are very close to what was only science fiction few years ago. So, nowadays, we are as close as possible to be able to reach what Gelernter defined as "Mirror Worlds" [9]. These are software models of some chunk of reality that can mimic every change in real-time and host a massive number of users each with a different view of the mirror world. In 1991, this definition was as close to science fiction as was the definition of "Metaverse" by Neal Stephenson in 1992 [18], as a computer generated virtual space where people, represented by their avatars may interact in a distributed fashion. Or as was the definition of "Cyberspace" by William Gibson in 1984 [10] (though he had introduced the term in 1982 ) "A consensual hallucination experienced daily by billions of legitimate operators, in every nation, by children being taught mathematical concepts... A graphic representation of data abstracted from banks of every computer in the human system. Unthinkable complexity. Lines of light ranged in the nonspace of the mind, clusters and constellations of data. Like city lights, receding into the distance"

Mirror World, Metaverse, Cyberspace are not unrelated. Their analysis shows three main common dimensions related to (i) the way they are syncing between physical and virtual worlds, (ii) the agents (artificial or human) populating the worlds and cooperating/competing with each other to fulfill their goals, and (iii) the social relations and interactions taking place among the huge number of agents playing different roles in those interactions

Ten years ago, Weyns et al. formulated in [22] three categories of research challenges as a consequence of following the idea of thinking the environment of a Multiagent System as a first-class citizen: the first category relates to a proper formalization of "environment" making fully clear what is an agent and which element of an overall system is part of the environment. Based on this formal understanding, [22] expected the development of a classification of different kinds of environments also in relation to a corresponding taxonomy of application domains. The second category of challenges drives this further and deals with challenges in exploring the relation between agents and their environment. This can be done with respect to three dimensions: the (software) architectures of agents in relation to the environment, the protocols and laws that govern interaction between agents and their environment as well as between agents mediated by their shared environment and last but not least the constraints that the environmental topology imposes on the agents. The third category of challenges deals with advancing the findings of research addressing the first two categories of challenges into engineering environments - both design and implementation 
of environments. Over the years, many works have addressed those challenges, from a specialized formalization of the environment as active support of interaction [17] to agent platforms that combine support for complex environmental structures, complex agent reasoning and organization concepts [4] among other frameworks, platforms, and advanced applications as for example in the agreement technologies area[2]. In this collection, one can find many more examples about how research and application related to environments for MAS has been advanced during the last decade.

Ten years ago, despite of its initially wide approach from multiple perspectives, the focus was on a very restricted type of environments, mostly on the required components and infrastructures for MAS. Such environments were identified mostly as the "real" world or a set of non-agent applications. In this contribution, we widen the perspective on the environment for systematically considering also mixed forms. We identify, analyze, and characterize the interesting application domains for MAS paying special attention to the different kind of environments they are situated in and related to. Consequently, we take into account the three different (usual) dimensions allowing to characterize these kind of domains: the environment, the agent and the interaction perspective. The environment cannot be seen as either physical or software-based, but also mixtures of "worlds" must be considered. As a consequence, the characterization of agents must be more fundamental based on its nature (artificial or human). Interaction as the third dimension, may happen on different levels of complexity - richness and intensity - ranging from interaction from simply being situated in the same environment to addressing the question how the mixed environment impacts on agent behavior that is based/resulting in sophisticated sociability. In this contribution, we will not formally define those dimensions, but by discussing different application domains in terms of those three dimensions and analyzing how the environment can support them, we address the first category of challenges adapting the treatment of the environment to modern (and future) developments.

The rest of the paper is structured as follows: in next section, the three different dimensions that we have introduced to classify the application domains, are detailed, Section 3 presents the different domains commented in the paper, positioning them in the three commented dimensions. After that, the following section, deals with the different levels of support that the environment, as a first class entity, gives to the different parts of the space given by the three presented dimensions. Lastly, some conclusions and some glimpse at the future are commented.

\section{Analysis Dimensions}

To drive these considerations beyond visionary literature, we analyze existing proposals taking a multi-agent system perspective. We then considere three dimensions that are worth to compare and position each virtual environment domain with respect to the other: types of environment, types of agents interacting 
in these environments and types of interaction, taking place in the system. One can easily imagine that the possible types in each dimension are more than the ones presented in these not-so-far-away futuristic scenarios. Hereby the interaction among the entities is actually the decisive factor in what concerns the intensity, mode and richness of the resulting worlds: how the environment mediates the interaction, who may interact with whom along which relation or role. In the following, we characterize the types in each of these three dimensions. We then analyze existing approaches and domains along them in Sec. 3.

\subsection{Environment Dimension}

The dimension related to the environment is directly accessed by systems. It distinguishes physical from virtual environments. The physical environment refers to the environment which is part of our actual reality, in which we, as humans, interact. There is no clear definition of virtual environment in the literature. As we can see in the existing approaches, "virtual environment" may refer to "a high-end user-computer interface that involves real-time simulation and interaction through multiple sensorial channels" [5].

In this paper, virtual environment subsumes two definitions according to the reference that the virtual environment may have to another environment. While the simulated environment has a reference environment, the synthetic environment may not refer to any other environment [12].

- The simulated environment makes only sense as a mapping from a physical environment that it sufficiently precisely represents. Interaction in such environment between simulated agents refers to the way agents of the physical environment interact with the reference environment [11].

- On the contrary, the usefulness of a synthetic environment is not determined by how well it matches another reference environment. It is judged by how much fun it is to interact with such an environment or how well it supports interaction between humans, agents and other entities.

Thus, virtual environments not necessarily refer to a physical environment example, but focus on user interaction, immersion and imagination (the so called Virtual Reality Triangle or $i^{3}$ ).

Considering social networks, the need for characterizing "social" environments emerges. They form an abstraction from environments with explicit spatial dimensions by focussing on relations between agents. Space and spatial distance - real or virtual - plays no role. We do not explicitly handle this type of environment, but subsume it under synthetic environments as it may not just reproduce relationships between humans, but enable the establishment of new ones. Additionally it needs a matter for manifestation such as an environment created by facebook or similar.

From the analysis conducted in this section, as we can see in Table 1, the range of types for the environment dimension could be: physical, synthetic or simulated. Let's note that virtual subsumes both synthetic and simulated types. 


\subsection{Agent Dimension}

The second dimension to characterize the considered approaches is the nature of the agents interacting in the system. Traditionally, one may find descriptive values along "passive - active", "reactive, deliberative, cognitive", etc. According to the existing approaches, the types that we will use in this dimension fits to the range of the environment dimension. Hereby, we characterize only agents and let aside objects, resources that are part of the environment. Thus, possible types for an agent along this second dimension are: human, robot or digital. As humans, robots possess a (physical) body that allows physical interaction, i.e. interaction in the physical environment. Robots exist and interact primarily physically. There is a tendency for equipping them with displays for interaction with humans - e.g. the Giraff robot, which is basically "skype on wheel" (http://www.giraff.org). As in the environment dimension, digital subsumes simulated agent and synthetic agent.

Both types of agents point to an entity that exists in the digital world. Both may refer or not to an entity in the physical world (human or robot). Simulated agents can be Virtual Characters which are often humanoid. Believability of their behavior is an important measure for their quality. Synthetic agents are created for a particular aim - for example a fully digital playing partner for children such as a Tamagotchi. In an extreme form, a synthetic agent may not be explicitly embodied, but may e.g. deliberately display information at a particular location in the virtual world. The overall system may contain different forms of agents at the same time.

Thus, we may identify the range of types for the agent dimension: human, robot or digital. Let's note that here also digital subsumes both synthetic and simulated types.

\subsection{Interaction Dimension}

As for agents, characterizing "interaction" is also not done for the first time. See for instance a quite comprehensive example in [8] of kinds of interaction in terms of relations, protocols or organizations that are proposed in the MAS domain. Knowing that background, we are aware of the simplification when we just consider how intensive and on what level of abstraction agents interact with each other and with their shared environment. We identify in the existing approaches a type that we denote as indirect (or stigmergy) in which each agent just interacts with its environment. For reasons of limited space, we use this term "indirect" to refer to any kind of interaction based on the use of the environment. The next value is what we call direct or message passing interaction that concern the exchange of messages between agents. In principle, we call social the other extreme type in this dimension: one agent knows and intensively interacts with a large set of other agents, if not all others, being aware (and maybe participating) to the social dimension (e.g. organisation) sustaining the agents participating to its environment. 
The set of types for the interaction dimension comprises thus: indirect, direct, social. As can be noticed, this dimension is of a different nature than the two other dimensions. This dimension doesn't take value in terms of reality or virtuality. It characterizes the existence or non existence of interaction between the entities to interact in the shared environment. However, we still can identify references to the real world in the sense that the kind of interaction that could be installed among the agents can refer or not to some kind of interaction that may exist among the humans or robots that they are simulating. In environments in which humans are immersed, at least the humans may not be able to interact ignoring the social context in which they are embedded. There is clear relation between complexity of an agent and complexity of the interaction that this agent may come up. The first two - interaction based on environment manipulation (indirect) and based on message exchange (direct) - form lower level characterizations based on the mean of interaction. The category of social may be technically reduced to those two forms, nevertheless we assume that this categorization is admissible focusing on the intensity and richness of interaction.

\section{Domain Classification}

In this section, we structure our analysis of the domains according to the environment dimension presented in the previous section. This organisation is just here to stress the fact that domains follow a smooth path from physical to virtual types. The following table (cf. Tab. 1) shows a representation of the three dimensions presented above, locating in the 3D corresponding space several application domains where multi-agent systems can be/are used. In this section, we use the coordinate system $(\mathrm{a}, \mathrm{e}, \mathrm{i})$ to locate each of the corresponding domains. When a coordinate is equal to 'all', we mean that all the values of the coordinate can be considered. After a tabular overview, we shortly justify our characterizations.

\subsection{Domains situated in Physical Environment}

Pure physical reality domains ( $\mathrm{e}=$ physical $-\mathrm{a}=$ human $-\mathrm{i}=$ all) refers to the "ordinary" real world in which humans are living, that means a domain where humans intensively interact with each other (i.e. interaction type of sociability embraces all values of this dimension) and the physical environment.

Robotic domains ( $\mathrm{e}=$ physical $-\mathrm{a}=$ robot $-\mathrm{i}=$ all) refers to domains where only robots populate the relevant sectors of the physical environment. Interactions may take all three forms. This might be surprising on the first sight, as with applications consisting of multiple robots often swarm robots are associated. These swarm multi-robot systems form examples based on intensive, yet simple stigmergic interaction. An impressing example is the Swarmanoid project [14], in which a robot swarm's task is to locate and fetch a particular book from a high shelf. Each robot can just perform simple tasks, but based on intensive, carefully designed stigmergic interaction the overall swarm could achieve the 


\begin{tabular}{|l|l|l|l|}
\hline Domain & Environment & Agent & Interaction \\
\hline \hline Pure physical reality & physical & human & all \\
\hline Robotic & physical & robot & all \\
\hline Humans-Robots System & physical & human, robot & all \\
\hline \hline Ambient intelligence & physical, synthetic & human, robot & all \\
\hline Data-Driven Simulations & physical, simulated & $\begin{array}{l}\text { human, robot, digital } \\
\text { agent }\end{array}$ & all \\
\hline Situated Multi-Agent Systems & physical, virtual & digital agent, robot & all \\
\hline Augmented Reality & physical, virtual & human & all \\
\hline Mirror World & physical, virtual & $\begin{array}{l}\text { human, digital agent, } \\
\text { robot }\end{array}$ & all \\
\hline Multi-Agent Based Simulations & simulated & digital agent & all \\
\hline Interactive Simulations & virtual & human, digital agent & all \\
\hline Virtual Reality & virtual & human, digital agent & all \\
\hline Social Networks & synthetic & human \\
\hline Table 1. Dooll
\end{tabular}

Table 1. Domains classifications according to Environment, Agent and Interaction dimensions. Let's note that even if the right column appears to be redundant as it has the same value for all rows, we intentionally kept it here as it is not intuitively clear that all domains contain all forms of interactions.

task. One may also find complex, social interactions in robotic domains: for example in RoboCup applications multiple robots form complex organizations with corresponding interactions for achieving a shared goal. This can be clearly characterized as social interaction.

Humans-Robots System are domains (e=physical $-\mathrm{a}=$ human, robot $-\mathrm{i}=$ all) where humans may participate in shared activities or otherwise interact with robots. This raises challenges for overall system design as the autonomous robots may need to be aware of what humans are doing (for a general overview of human activity recognition see [1]). Interaction with one human for shared activity works quite well, interacting with multiple humans is still a challenge.

\subsection{Domains situated in Physical-Virtual Environment}

Ambient Intelligence is a domain ( $\mathrm{e}=$ physical, synthetic $-\mathrm{a}=$ human, robot $\mathrm{i}=$ all) where the environment is real and the agents are human and robots. An important form of robots for this kind of domain are sensors. No matter whether they are mobile or stationary, sensors are physical hardware placed in the physical world. Thus, we subsume them under robotic agents. Sensors may be embedded into a control loop for regulating environmental features, or may be organized in sensor networks for producing complex information necessary to support human activity or well-being. In this domain, the task of the robots is to support the human by adapting the environment and providing access to different functionalities in it, by eventually interacting through a synthetic 
environment. Examples are agents in charge of adjusting temperature and light intensity for individual humans. Depending on the task and the number of agents, there might be intensive interaction between the agents, but the main focus is on supporting the human.

Data-Driven Simulations refers to domains (e=physical, simulated - a=human, robot, digital agent $-\mathrm{i}=$ all) that connect simulation to reality based on sensor data integrated during simulation runtime. Thus, environment is basically real, but simulations are done for extrapolating the current environmental state, for example for supporting decision making about the current state. An example could be found in the OLSIM ${ }^{5}$ system: the highway network of North-Rhine Westfalia - a densely populated area in Germany - is connected to a simulation of relevant highway segment via sensors that count vehicles entering those highways. Every time a vehicle enters a ramp an agent is generated in a simulation and vice-versa for agents leaving the segment. A traveler can access the simulated (predicted) congestion via Internet for making decisions about routing. As for all kinds of simulations, the reproduced interaction may eventually range from simple to complex.

Situated Multi-Agent Systems as a domain that is established ( $\mathrm{e}=$ physical, virtual $-\mathrm{a}=$ digital agent $-\mathrm{i}=$ all $)$ and concerns all those multi-agent systems operating in some physical environment, where a virtual environment is introduced in order to provide some functionality concerning either the agent access to the physical layer, or agent coordination. A main example is the decentralized control of AGV case proposed by D. Weyns et al. [21].

Augmented Reality (e=physical, virtual - a=human - $\mathrm{i}=$ all) is characterized by an environment, but populated with human agents and artificial ones. [15] introduced the term "Mixed Reality" to describe a continuum of environments from fully physical via augmented reality and augmented virtuality to fully virtual environments. In Augmented Reality the main part of the environment is real. This mix of virtual and physical environment enables new forms of interaction in the overall system mixing for example haptic experiences or smells with virtual information displays. This is interesting not only for entertainment (see for example the INVIZIMALS ${ }^{6}$ game), but also information services depending on physical location.

Mirror Worlds (e=physical, virtual - a=human, digital, robot - i=all) - as defined in [7] - can be conceived as an agent-based extension of augmented and mixed reality. Both human and artificial agents inhabit an environment which is both physical and augmented of a digital virtual layer (the mirror), coupled to the physical one. Mirroring is given by the fact that physical things, which can be perceived and acted upon by humans in the physical world, have a digital

${ }^{5}$ http: //www.autobahn.nrw.de/index_e.html

${ }^{6}$ http://invizimals.eu.playstation.com/ 
counterpart (or augmentation, extension) in the mirror, so that they can be observed and acted upon by agents. Vice versa, an entity (artifact) in the Mirror World that can be perceived and acted upon by software agents may have a physical appearance (or extension) in the physical world - e.g. augmenting it, in terms of Augmented Reality - so that it can be observed and acted upon by humans (by means of e.g. smart-glasses). This implies a form of coupling, such that an action on an object in the physical world causes some kind of changes in entities in the mirror, perceivable then by software agents. Vice versa an action by agents on an artifact in the Mirror World can have an effect on things in the physical world, perceivable by people.

\subsection{Domains situated into Virtual Environment}

Multi-Agent Based Simulations is a well established domain ( $\mathrm{e}=$ simulated $\mathrm{a}=$ digital agent $-\mathrm{i}=$ all) in the research community. It clearly forms an extreme domain since environment is virtual (i.e. simulated), agents are artificial (i.e. simulated), interactions are defined following observable or hypothesed interactions in the given reference system possibly embracing all three values of this dimension.

Interactive Simulations refers to a domain (e=virtual - a=human, digital agent - $\mathrm{i}=$ all) that generalizes from the pure multi-agent simulation approaches and raises multiple sub-domains: the environment is virtual, one or more agents may be human, others may be artificial. The environment may refer to the real world or may be completely synthetic. Interaction between humans and simulated entities may happen on different levels of immersion. A human may perform a simulated biological experiment or may be immersed in a flight simulator. Usually some (more or less realistic) physics are simulated, capturing how the environment (including other agents) reacts to user actions. In multiagent simulation, participatory approaches (such as [3]) play a more and more important role mainly in cases in which reliable empirical data is missing for model development. Another motivation for immersing humans into multi-agent simulations can be found if stakeholders should be supported in learning about possible system responses. Hereby, human stakeholders are involved or even immersed in role-playing game-like simulations, yet those approaches mainly .

Virtual Reality defines a domain (e=virtual - $\mathrm{a}=$ human, digital agent $-\mathrm{i}=$ all) that is nearby interactive simulations and multi-agent based simulations: the environment is virtual, it may include humans and artificial agents - more humans than in multiagent-based simulations and interactive simulations. The environment may refer more to a real environment than in interactive simulations, yet without the need of reproducing a particular original system. Again, we include also here entertainment simulations (e.g. games, "Second Life" where humans interact with other humans and artificial agents in a shared immersive virtual environment). This domain includes not only the Metaverse of Neal Stephenson, but also the Cyberspace of William Gibson. The original idea was to differentiate 
between Virtual Reality and Cyberspace, by having the former as an individual experience, and the last as a social one. We selected "virtual" as the value for the environment capturing both synthetic and simulated - so the environment in the Virtual Reality domain may have a reference to a real environment or not, however the stringency of connecting the environment to an original one may not be as strong as in the simulation applications. Here, whether the environment is interesting or believable is more important than its validity.

Social Networks form a domain ( $\mathrm{e}=$ virtual $-\mathrm{a}=$ human $-\mathrm{i}=$ all) where social relations are manifested. It is the facebook / twitter environment in which virtual space is abstracted into a (dynamic) network of relationships. Social reality might be combined with synthetic environments or analyzed with simulated environments. Yet it is something clearly different from virtual environments as it maps explicit real relations ("follows", "friend-of") into a artificial structure and provides meeting opportunities that are potentially decoupled from concurrent behavior/interaction. It could be seen as a manifestation of social interactions.

\section{Levels of Support by the Environment}

In the global picture built in the previous section showing the importance of the environment in each of the analyzed domain, the level of support of this environment as a first-class entity can be different depending on the specific point or subspace that we consider. In general, three main support levels can be identified [20]:

- deployment support level, which is the simplest level in which the environment just introduces a notion of action, perception, observability, without any kind of modularisation;

- abstraction support level, where the environment introduces first class logical abstraction to modularize actions/perceptions and to encapsulate functionalities;

- interaction mediation support level, where the environment has a role in enabling and ruling/governing/mediating the interaction and communication among the agents.

\subsection{Levels of support for digital agents or robots}

In literature, these levels have been identified and used to analyse mostly systems considering only artificial agents on the agent type dimension. In our picture, relevant examples for that case are:

- pure virtual environment, digital agents only - This is the case of agentbased and MAS-based simulation. Here the virtual environment has the fundamental role of modelling the space of interaction among agents, at the proper level of abstraction. 
- physical + virtual environment, digital agents + robots - This is the case of situated MAS, which is one of the reference cases on which the levels in [20] have been defined. As widely discussed in literature, the integration of physical and virtual environment can be effectively exploited to support in particular agent coordination.

\subsection{Levels of support for human agents}

The role of the environment support for systems considering only human agents on the agent type dimension has been discussed in the context of cognitive sciences and human socio-psychological fields. Relevant examples in our picture are:

- physical environment, human agents - This is the pure physical reality case. In this case, Activity Theory and Distributed Cognition focus the importance of physical objects and tools in supporting human activities and problem solving. Even if it is not about the agent environment, the three levels can be adopted as well. The deployment levels refer to those cases in which the physical environment is not specifically designed in order to support human activities; it is just used as the neutral place where some activity takes place. The abstraction support level occurs when the physical environment is specifically conceived and designed to mediate and support human activities; an example is given by any kind of tool (e.g. a hammer) used to do some kind of job. The interaction mediation support level happens when the design is explicitly conceived to help the cooperation and communication of humans. Examples include blackboards, cell phones, post-its, etc.

- virtual environment, human agents - This is the case of Social Networks, for instance. In this case, the tools depicted in the previous point are greatly enhanced by the availability of the information technology, that makes it possible to create more powerful social media that allow to augment human communication and interaction besides time-space barriers. As a distinguishing feature, these tools implement mechanisms to make communication indirect and persistent, so as to create emergent/self-organizing/stigmergic form of coordination and cooperation.

- physical + virtual environment, human agents - This is the case of Augmented Reality. The integration of the physical environment has the effect to strongly couple and ground the first-class environment abstraction layer with physical artifacts of the reality, augmenting their functionalities. Or, to situate the virtual entities defined in the virtual environment in some physical location. This can be exploited to define a whole new space of spatially-based functionalities and services, as those that are typically provided by mobile augmented reality applications [16].

\subsection{Levels of support for human, digital or robot agents}

Finally, a less explored subspace - in particular in the environments for MAS literature - concerns those cases in which human and artificial agents (robot or 
digital ones) are both characterising the agent type dimension. Two main cases are the following:

- virtual environment, human + digital agents - This includes Virtual Reality as well as Intelligent Virtual Environments [5], where virtual environments are inhabited by both humans - represented by some kind of avatars - and artificial agents, both perceiving and acting on the same shared environment. The virtual environment in this case provides functionalities in terms of abstraction, by allowing human users to physically represent and perceive virtual entities, which may be designed to encapsulate different kinds of functionalities, services. Besides, these entities can be exploited also to ease the communication/coordination among avatars, in particular with other human users that may be physically located in a different place, as well as with purely digital agents part the same world.

- physical + virtual environment, human + digital agents - This is the case of Mirror Worlds and the environment support can be conceived by integrating synergically what discussed for Augmented Reality and situated MAS. In this case, the abstraction support level provided by the environment is twofold: from the artificial agents point of view, it provides a way to represent, perceive and interact with physical things, represented and abstracted by artifacts; from the human agents point of view, the virtual environment provides a way to augment the physical world with further functionalities, as well as to empower humans with further cognitive/sensing/acting capabilities. The interaction mediation support level in this case allows for designing environment-meditated coordination and cooperative strategies possibly self-organising, emerging - that exploit both the physical and digital layer, towards new forms of Behavioural-Implicit Communication and stigmergy $[6,19]$.

\section{Conclusions or what is waiting ahead of us}

In the last years, there has been a growing importance of the environment as a first class entity in the developing of MAS. The increasing maturity of more and more feasible and advanced technology in the area of Virtual Reality has lead to a growing interest in Virtual Environments in both, society and research. As a reflection of this, one can also observe an increasing number of applications of MAS using this technology creating and enabling new environments for the different forms of MAS.

In this chapter, we have extended the idea of the environment as a first class entity for explicitly integrating Virtual Environments and as a consequence mixing the context that they may provide with other types of environments, physical, simulated or synthetic. Before discussing what the explicit treatment can offer for those application areas, we had to locate them clearly in an overall conceptual framework widening the perspective beyond the original environment for multiagent systems idea. From that point of view we analysed environments in 
different forms together with the overall system application context. We characterized the latter in terms of types of agents and richness and intensity of interaction. We classified various examples of application domains along those dimensions indicating how the environment impacts the overall setup. The contribution of this chapter can be seen in the clear characterization of systems with a wider perspective beyond environments for agents. In the same way software agents, robots and humans can interact in one environment also environments for those diverse and heterogeneous multiagent systems can be of different (also mixed) types. Having a look at science fiction literature and the environment concepts authors have foreseen, may serve as a source of inspiration for setups that once were visionary, but now become more and more reality.

Starting from our discussion in section 4 on what level of support can be expected from the environment in the relevant coordinates/cases, future work in this research area will focus on developing not only frameworks for implementing these kind of applications. Formalized, unifying meta-models, methodologies, and eventually developing toolkits to support the designer to create and manage these applications will clearly help to enable future useful applications beyond what we are able to realize now.

\section{Acknowledgments}

This work is partially supported by the Spanish government grant MINECO/FEDER TIN2012-36586-C03-01 and Valencian Government Project PROMETEOII/2013/019.

\section{References}

1. Aggarwal, J.K., Ryoo, M.S.: Human activity analysis: A review. ACM Comput. Surv. 43(3), 16:1-16:43 (2011)

2. Argente, E., Boissier, O., Carrascosa, C., Fornara, N., McBurney, P., Noriega, P., Ricci, A., Sabater-Mir, J., Schumacher, M., Tampitsikas, C., Taveter, K., Vizzari, G., Vouros, G.: The Role of the Environment in Agreement Technologies. AI Review pp. 1-15 (2013)

3. Barreteau, O., et al.: Our companion modelling approach. Journal of Artificial Societies and Social Simulation 6(1) (2003)

4. Boissier, O., Bordini, R.H., Hübner, J.F., Ricci, A., Santi, A.: Multi-agent oriented programming with jacamo. Science of Computer Programming 78(6), 747 - 761 (2013)

5. Burdea, G., Coiffet, P.: Virtual Reality Technology. John Wiley and Sons, New Jersey, US (2003)

6. Castelfranchi, C., Pezzullo, G., Tummolini, L.: Behavioral implicit communication (BIC): Communicating with smart environments via our practical behavior and its traces. International Journal of Ambient Computing and Intelligence 2(1), 1-12 (Jan-Mar 2010)

7. Castelfranchi, C., Piunti, M., Ricci, A., Tummolini, L.: Ami systems as agent-based mirror worlds: Bridging humans and agents through stigmergy. In: Bosse, T. (ed.) Agents and Ambient Intelligence, Ambient Intelligence and Smart Environments, vol. 12, pp. 17-31. IOS Press (2012) 
8. Ferber, J.: Multi-Agent Systems: An Introduction to Distributed Artificial Intelligence. Addison Wesley Longman (1999)

9. Gelernter, D.: Mirror worlds - or the day software puts the universe in a shoebox: how it will happen and what it will mean. Oxford University Press (1992)

10. Gibson, W.: Neuromancer. Ace (1984)

11. Kluegl, F., Fehler, M., Herrler, R.: About the role of the environment in multi-agent simulations. In: Weyns, D., Dyke Parunak, H., Michel, F. (eds.) Environments for Multi-Agent Systems, Lecture Notes in Computer Science, vol. 3374, pp. 127-149. Springer Berlin Heidelberg (2005)

12. Krueger, M.: Artificial reality II. Addison-Wesley (1991)

13. Luck, M., Aylett, R.: Applying artificial intelligence to virtual reality: Intelligent virtual environments. Applied Artificial Intelligence 14(1), 3-32 (2000)

14. M. Dorigo, D. Floreano, L.M.G., et al.: Swarmanoid: a novel concept for the study of heterogeneous robotic swarms. IEEE Robotics \& Automation Mag. pp. 60-71 (2013)

15. Milgram, P., Kishino, A.F.: Taxonomy of mixed reality visual displays. IEICE Transactions on Information and Systems pp. 1321-1329 (1994)

16. Olsson, T., Salo, M.: Online user survey on current mobile augmented reality applications. In: Proceedings of the 2011 10th IEEE International Symposium on Mixed and Augmented Reality. pp. 75-84. ISMAR '11, IEEE Computer Society, Washington, DC, USA (2011)

17. Saunier, J., Balbo, F., Pinson, S.: A formal model of communication and context awareness in multiagent systems. Journal of Logic, Language and Information 23(2), 219-247 (2014)

18. Stephenson, N.: Snow Crash. Bantam Books (1992)

19. Tummolini, L., Castelfranchi, C.: Trace signals: The meaning of stigmergy. In: Weyns, D., Parunak, H.V.D., Michel, F. (eds.) Environments for MultiAgent Systems III, LNAI, vol. 4389, pp. 141-156. Springer (May 2007)

20. Weyns, D., Omicini, A., Odell, J.: Environment as a first class abstraction in multiagent systems. Autonomous Agents and Multi-Agent Systems 14(1), 5-30 (2007)

21. Weyns, D., Schelfthout, K., Holvoet, T., Lefever, T.: Decentralized control of e'gv transportation systems. In: Proceedings of the fourth international joint conference on Autonomous agents and multiagent systems. pp. 67-74. ACM (2005)

22. Weyns, D., Schumacher, M., Ricci, A., Viroli, M., Holvoet, T.: Environments in multiagent systems. Knowledge Engineering Review 20(2), 127-141 (2005) 